\title{
Advancing Chip-Based Gas Composition Sensors for Efficient Planetary Exploration
}

\author{
Planetary Decadal Survey White Paper \\ 15 September 2020
}

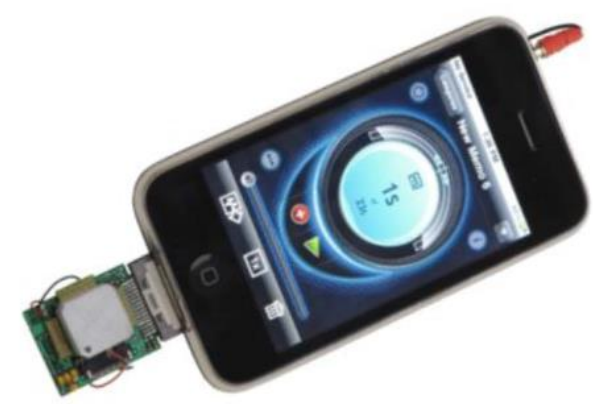

Authors

Michael H. Wong (UC Berkeley, SETI Institute)

+1-510-224-3411

mikewong@astro.berkeley.edu

Hossain Mohammad Fahad

(UC Berkeley, Serinus Labs, Inc.)

\author{
Jing Li \\ (NASA Ames) \\ Kunio Sayanagi \\ (Hampton Univ.) \\ Paul Steffes \\ (GA Tech) \\ Sushil Atreya \\ (Univ. Michigan)
}




\section{Executive summary}

Chemically-sensitive transistors can enable gas-composition measurements with minimal volume, mass, and power requirements, for space applications such as in-situ exploration of planetary atmospheres, cached sample monitoring, and crew health and safety. Although chipscale gas sensors have some limitations compared to mass spectrometers, their smaller resource requirements would enable composition measurements aboard a much wider range of space missions. We present two specific applications that need this technology: miniaturized atmospheric entry probes at the ice giants, and high-cadence measurement of Martian $\mathrm{H}_{2} \mathrm{O}, \mathrm{O}_{2}$ and $\mathrm{CH}_{4}$ variability. Many other applications could also be enabled by these sensors. We recommend (1) that chip-based composition sensor technology be advanced to reach the high sensitivities needed to achieve solar system measurement requirements, (2) that flight instruments be developed based on these sensors, and (3) that tools be supported to increase discoverability of instruments for solar system exploration (both flown and under development) and facilities for instrument development and testing (such as planetary atmosphere simulators). These tools could be coordinate similarly to a NASA PDS node.

\section{Motivation}

Initial in-situ measurements of atmospheric composition in planetary atmospheres such as Venus, Mars and Jupiter have been achieved using mass spectrometers (Oyama et al. 1977, Niemann et al. 1998, Mahaffy et al. 2013, Johnson and de Oliveira 2019) and a tunable laser spectrometer on Mars (Webster et al. 2013). Analysis of the results has revealed surprises in terms of vertical and temporal variability in key species indicative of physical and chemical processes in the atmosphere and surface (Niemann et al. 1998, Webster et al. 2018, Trainer et al. 2019). The observations have opened new questions requiring measurements at the ice giants in addition to Jupiter, and at higher temporal sampling rate at Mars. Chip-based composition sensors are well suited to these problems, because their low requirements for volume and power enable them to be incorporated into payloads where a mass spectrometer could not be supported, or as an auxiliary payload on smallsats or cubesats to provide wider coverage. These sensors are well-suited to additional applications in the areas of sample caching/return and human spaceflight. The light weight and low power requirements of these sensors would enable clusters of sensor suites to perform area measurements and map chemical profiles.

\section{Chip-based composition sensor approaches}

Chemically-sensitive field-effect transistors (FET) are good because they operate at ambient pressure/ temperature, are very compact, and require small amounts of power. The sensors respond to sample gases that modulate channel current in the linear amplifying region of a special transistor. Chemically-sensitive materials are used in the gate to induce a threshold voltage shift when the sensor encounters the target gas due to the interaction occurring between the gate materials and the target gas molecules. Chemical sensitivity has been demonstrated for gate materials including conducting polymers, nanomaterial composites and metal nanowires. 
Chemiresitor is another chip-based approach. NASA ARC has developed a chemical sensor array for trace chemical detection in the past 16 years. The sensor technology is under continued development, and it has been used to detect $\mathrm{CH}_{4}, \mathrm{NH}_{3}, \mathrm{H}_{2} \mathrm{~S}$ and $\mathrm{H}_{2} \mathrm{O}$ in ambient conditions (Li et al. 2003; Lu et al. 2004; Hannon et al. 2014, 2016). The working principle of the sensor is shown below in Figure 1. The sensor heads contain nanostructured materials such as single-walled carbon nanotubes (SWCNT) that are differently functionalized/doped/coated to create sensitivities to different gas molecules. Figure 1 shows an example of a chemiresistor prototype with doped SWCNT, with sensor response to ammonia.

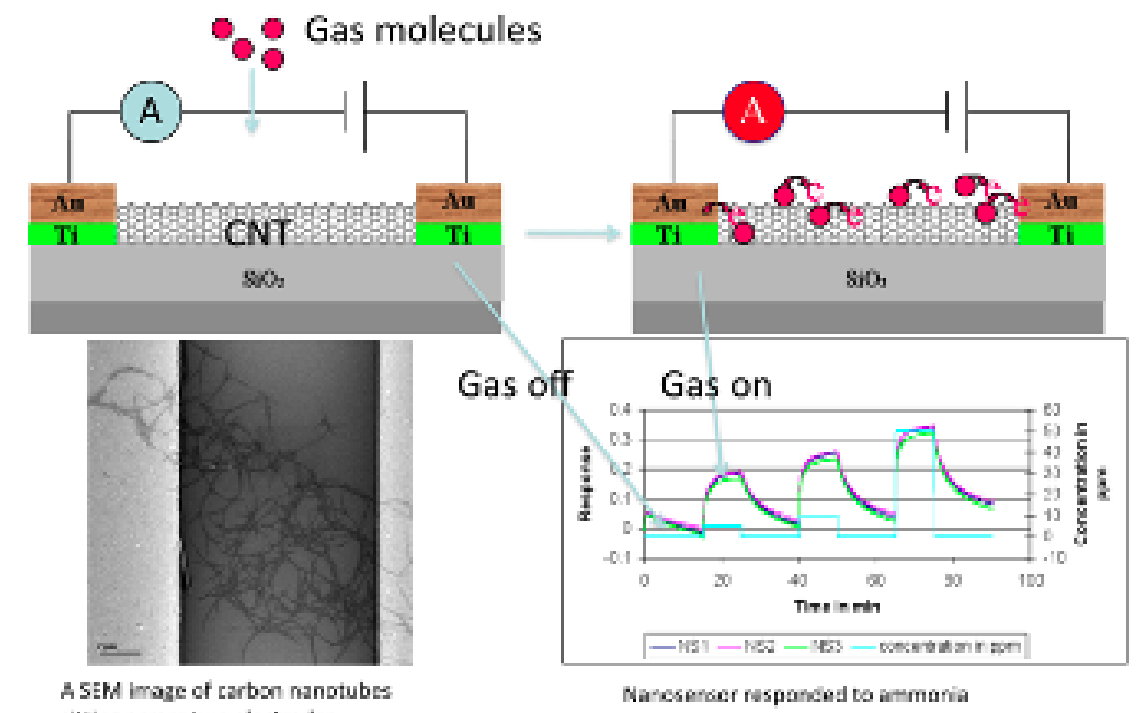

FIGURE 1 - Chemiresistor chip sensor working principle. Top: Target gas molecules interact with a chemically-sensitive resistor material (doped SWCNT reacting to $\mathrm{NH}_{3}$ in this case).

Bottom left: Scanning electron micrograph of the top of the sensor, with carbon nanotubes. Bottom right: Gas concentration is measured by changes in sensor resistivity.

Chip-based sensors enable new classes of planetary atmospheric in-situ exploration because of the low power required (nanowatt range) and the low volume occupied (several $\mathrm{cm}^{3}$, including electronics), especially compared to traditional instrumentation such as mass spectrometers, which include vacuum-pumping systems. Although an individual transistor may be sensitive to a single target gas over a specific concentration range, multiple transistors are included on a single chip to span multiple target gases and to expand sensitivity ranges. After each measurement cycle, sensors can quickly recover after reset procedures such as microheater activation or UV flashing (Fahad et al. 2017, Sayanagi et al. 2020).

\section{Performance characterization: sensitivity, specificity}

High sensitivity (the minimum target gas partial pressure detectable by a sensor) is needed to measure trace gases. A very wide parameter space can be explored to achieve the necessary sensitivity by modifying the chemically-sensitive transistor gate material. For example, singlewalled carbon nanotubes can be doped with gold nanoparticles to increase sensitivity to $4.6 \mathrm{ppb}$ from to $40 \mathrm{ppb}$ for $\mathrm{NO}_{2}$ detection (Young et al. 2005). Table 1 lists the highestsensitivity achieved for some example target gases by various sensors. 
High selectivity (the ability of the sensor to respond exclusively to the target gas) is needed to measure species in a gas mixture. Banks of sensing heads (Fig. 2A) can include a range of functionalized nanostructured materials, identifying species in gas mixtures via chemical interactions between the sensing materials and the target gas molecules. Pattern recognition techniques can enhance multi-chemical detection and selectivity. As with sensitivity, variations in transistor gate material are exploited to achieve the desired selectivity targets.

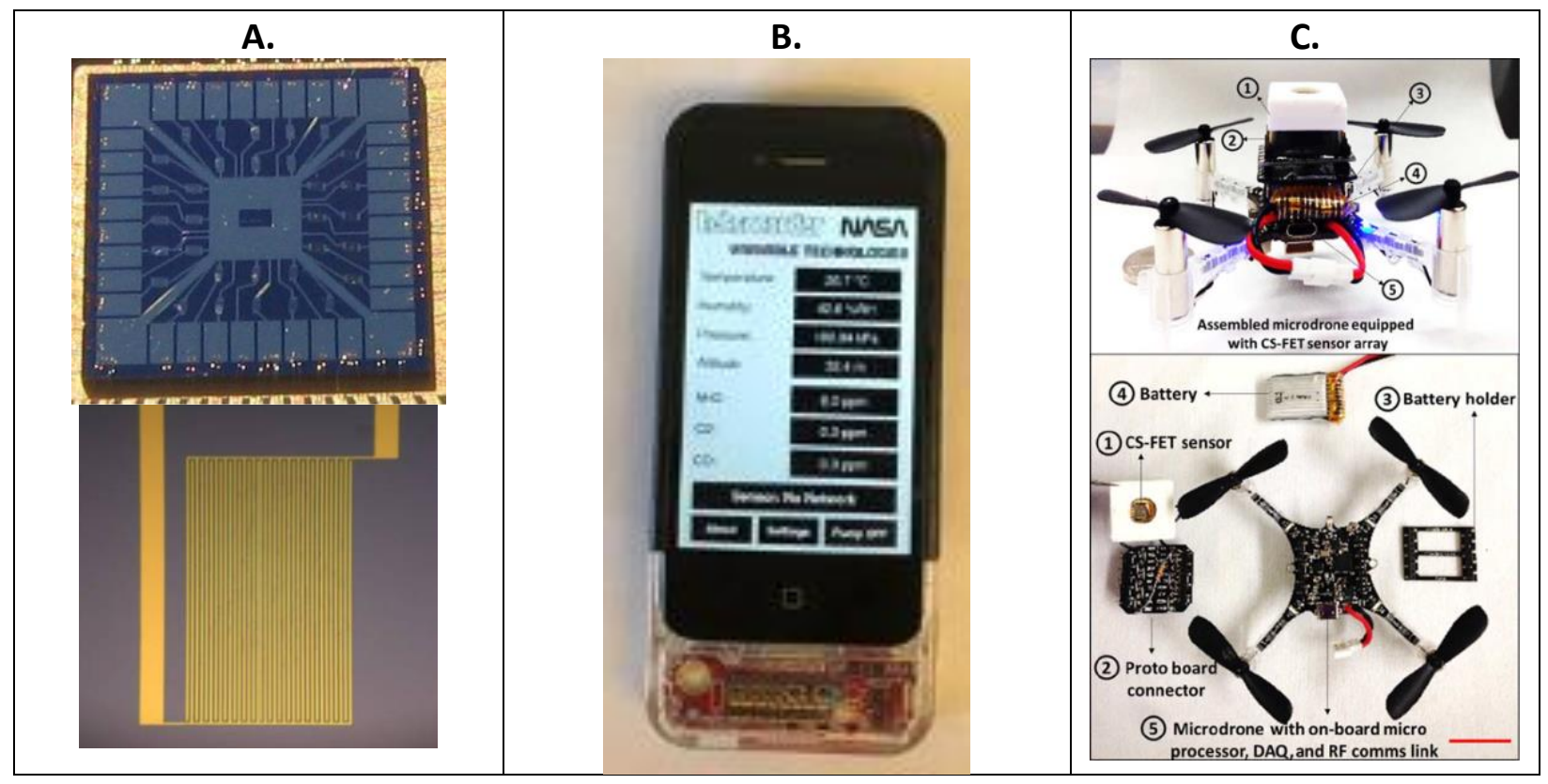

FIGURE 2 - Chip sensor implementations. A. Top: A sensor array with 32 sensing heads on a single $1 \mathrm{~cm} \times 1 \mathrm{~cm}$ chip. Bottom: A close-up of a sensing head showing gold electrodes bridged by doped single-walled carbon nanotubes (Li et al. 2003). B. A composition sensor attached to an iPhone 4 for power and data acquisition (Li et al. 2012). C. A microdrone equipped with a $\mathrm{Ni}$ Pd-based $\mathrm{H}_{2}$-sensitive composition sensor (Fahad et al. 2017).

TABLE 1 - Demonstrated sensitivity levels of CS-FET sensors, for target gases in a background of air. Testing was at STP, except for $\mathrm{H}_{2} \mathrm{O}$ in Mars conditions (6 mbar total pressure, $-45^{\circ} \mathrm{C}$ ).

\begin{tabular}{|l|l|l|l|l|}
\hline $\begin{array}{l}\text { Target } \\
\text { gas }\end{array}$ & $\begin{array}{l}\text { Sensitivity } \\
\text { (concentration) }\end{array}$ & $\begin{array}{l}\text { Sensitivity } \\
\text { (partial } \\
\text { pressure) }\end{array}$ & Sensor type & References \\
\hline $\mathrm{CH}_{4}$ & $1 \mathrm{ppm}$ & $1 \mu \mathrm{bar}$ & CNT & Lu et al. (2004) \\
\hline $\mathrm{H}_{2} \mathrm{~S}$ & $1 \mathrm{ppb}$ & $1 \mathrm{nbar}$ & Pd-Au on $3.5 \mathrm{~nm}$ Si FET & Fahad et al. (2017) \\
\hline $\mathrm{NH}_{3}$ & $0.5 \mathrm{ppm}$ & $500 \mathrm{nbar}$ & CNT & Hannon et al. (2016) \\
\hline $\mathrm{O}_{2}$ & $0.06 \%$ & $0.6 \mathrm{mbar}$ & Graphene & Li et al., in prep. \\
\hline $\mathrm{H}_{2} \mathrm{O}$ & $10 \mathrm{ppm}$ & $0.06 \mathrm{nbar}$ & CNT & Hannon et al. (2014) \\
\hline $\mathrm{H}_{2}$ & $0.3 \%$ & $300 \mathrm{mbar}$ & Ni-Pd & Fahad et al. (2017) \\
\hline $\mathrm{CO}$ & $0.5 \mathrm{ppm}$ & $500 \mathrm{nbar}$ & Sulfonated CNT & Hannon et al. (2014) \\
\hline
\end{tabular}




\section{Example science application 1: Ice giants mini descent probe}

Cloud-forming gases in ice giant atmospheres include $\mathrm{CH}_{4}, \mathrm{NH}_{3}$, and $\mathrm{H}_{2} \mathrm{~S}$. Their concentrations vary vertically due to condensation (Fig. 3), advection, and diffusion, so measuring cloudforming gases directly-even at $\mathrm{P}<5-10$ bar-will deliver major new insights into the weather and climate on these planets, and physics on scales from micro to planetary (Guillot et al. 2020, Tollefson et al. 2019, Hueso and Sánchez-Lavega et al. 2019). Although the bulk abundances of these volatile species have implications for planet formation (Atreya et al. 2020), it is possible that $\mathrm{CH}_{4}$ is the only volatile with a measurable bulk abundance, near 1-2 bar. Deeper than the $\mathrm{CH}_{4}$ ice cloud base, remote-sensing evidence has been accumulating that $\mathrm{H}_{2} \mathrm{~S}$ is the next-most abundant cloud-forming gas, not $\mathrm{NH}_{3}$ as may be expected from solar composition (Gulkis et al. 1978, de Pater et al. 1991, Irwin et al. 2018; 2019). The precise vertical profile of $\mathrm{H}_{2} \mathrm{~S}$ concentration may solve the mystery of whether cloud chemistry or bulk composition is responsible for the massively non-solar N/S ratio near the $\mathrm{CH}_{4}$ cloud base ( 1 bar).

The Juno mission revealed highly unexpected deep compositional variation - in the horizontal direction as well as the vertical direction-on Jupiter, deepening the problems interpreting compositional data from the Galileo Probe Mass Spectrometer (Wong et al. 2004, Folkner et al. 1998, Ll et al. 2017). Miniaturized composition sensors enable multi-probe missions to study deep spatial variations such as those detected by Juno. The results would resolve discrepancies facing various remote-sensing studies, such as degeneracies between temperature and composition, or limited knowledge of absorption coefficients (Bjoraker et al. 2018, Sromovsky et al. 2011, Giles et al. 2017, Li et al. 2017). Secondary probes provide ideal answers to two questions: How do volatile gas profiles vary in different locations? What ground truth can anchor remote-sensing data on a global scale? To address these questions, a low-mass/volume secondary probe requires chip-based sensors (Sayanagi et al. 2020).

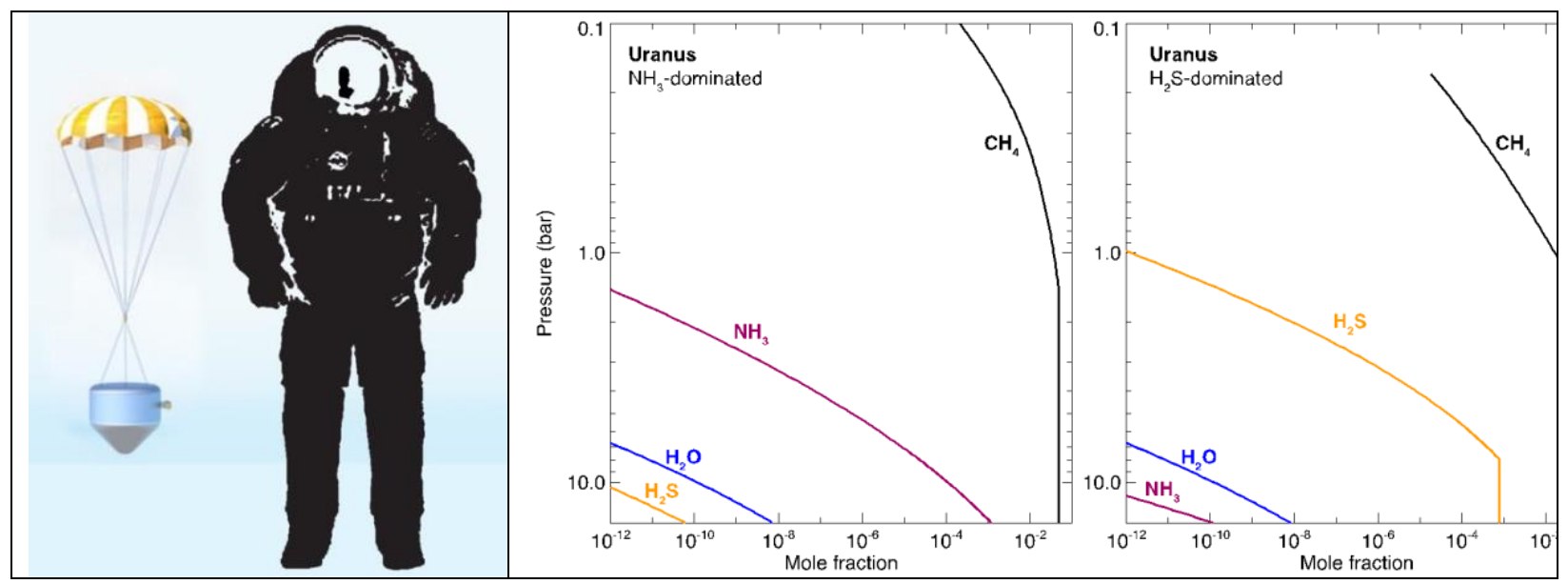

FIGURE 3 - Left: Chip-based sensors would enable a miniaturized atmospheric entry probe (30 $\mathrm{kg}$; shown to scale with an astronaut) to measure composition from 0.5-10 bar in Uranus (Sayanagi et al. 2020). Right: Saturated vapor pressure curves for Uranus set measurement requirements for in-situ confirmation of a supersolar $\mathrm{S} / \mathrm{N}$ elemental ratio, as suggested from radio, millimeter, and microwave remote sensing. 


\section{Example science application 2: Mars high-cadence variability}

The atmospheric composition of Mars was known to vary in response to polar cap condensation/sublimation in the $\mathrm{CO}_{2}$ cycle, but a range of investigations has demonstrated additional variability in two gases of major astrobiological interest: $\mathrm{O}_{2}$ and $\mathrm{CH}_{4}$ (Krasnopolsky et al. 2004, Formisano et al. 2004, Mumma et al. 2009, Korablev et al. 2019, McConnochie et al. 2019). Aside from the question of biogenic or geochemical origins, the observed variation may lead to advances in our understanding of habitability at the edge of our current understanding of the habitable zone (Yung et al. 2018, Stamenkovic et al. 2018). Both $\mathrm{CH}_{4}$ and $\mathrm{O}_{2}$ variations are far more rapid than their relevant photochemical timescales (Wong et al. 2003, Atreya and Gu 1994, Krasnopolsky 1993).

The frequency of $\mathrm{O}_{2}$ and $\mathrm{CH}_{4}$ measurements from the MSL SAM instrument (Fig. 4) is limited by the large power and thermal requirements for each measurement. These requirements place strong limits on the frequency of measurements, the simultaneity of $\mathrm{CH}_{4}$ and $\mathrm{O}_{2}$ measurements, and sampling of the diurnal cycle. All of these limitations could be overcome by a chip-based composition sensor, if sensitivity and specificity to $\mathrm{O}_{2}$ and $\mathrm{CH}_{4}$ can be enhanced to meet requirements. Exploring diurnal, seasonal, and inter-annual variability will require future measurements at much higher cadence (Moores et al. 2020).

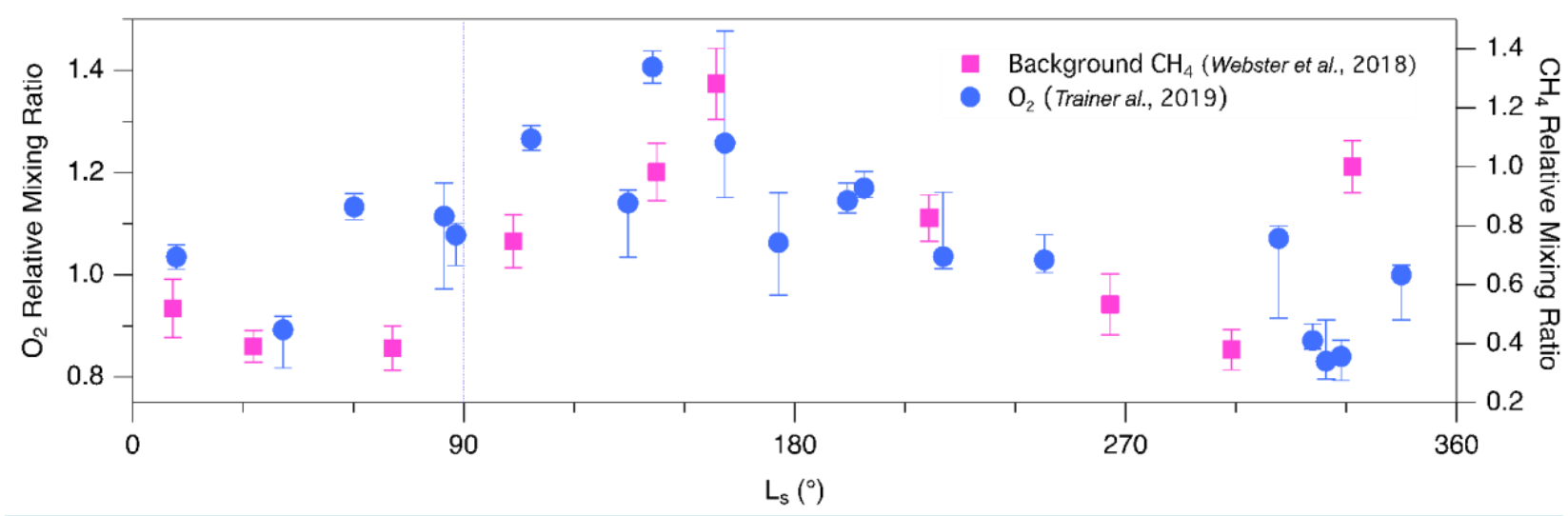

FIGURE 4 - Seasonal variation in the volume mixing ratios of $\mathrm{O}_{2}$ and $\mathrm{CH}_{4}$ cannot be explained by current models of atmospheric transport and photochemistry. Data shown here were measured over 4 Mars years by the mass spectrometer and tunable laser spectrometer on the Curiosity rover. The low overall temporal sampling rate is due to rover resource limitations. Chip-based composition sensors with the necessary sensitivity could obtain measurements at a higher cadence due to reduced power requirements. Data shown are relative to mean annual volume mixing ratios of 0.00153 for $\mathrm{O}_{2}$ and $2.13 \mathrm{ppb}$ for $\mathrm{CH}_{4}$ (Trainer et al. 2019, Webster et al. 2018).

\section{Additional applications}

- Caching and sample return are an important part of NASA's current and future activities. Some samples may potentially include volatile components, including samples from comets, Mars, and permanently shadowed craters on bodies such as the Moon or Mercury. Monitoring of these samples to characterize volatile loss during their 
collection, storage, and transport (Veverka 2010) could be efficiently conducted using chip-based composition sensors.

- In the human space flight arena, breathable air contamination could be monitored by chip-based composition sensors. This application has been demonstrated in settings such as oil-fields and refineries (Fahad et al. 2017), and chemiresistor sensors have been flown on the ISS. Both fixed (like a smoke alarm) and mobile (e.g., on a drone or mounted on a spacesuit) implementations would be of value.

- Chip-based composition sensors enable in-situ sampling at Venus, for balloons, descent modules, and landed assets. This application carries the additional challenge of hightemperature electronics (Glaze et al. 2018, Makel et al. 2020).

Development priority: increasing sensitivity for low concentrations. Table 2 provides an example, based on the SNAP study for a miniaturized atmospheric entry probe at Uranus. For $\mathrm{H}_{2} \mathrm{~S}$, existing sensors have already demonstrated the required sensitivity (Fahad et al. 2017). But for several gases, improvements to the chemically-sensitive transistor gate material are needed to measure target gases in the Uranus atmosphere. For example, sensitivity to $\mathrm{CH}_{4}$ must be increased by a factor of 10 for the Uranus/Neptune case, or a factor of $10^{6}$ for the Mars case. Sensitivity to $\mathrm{O}_{2}$ requires a 100x improvement for the Mars case. The ice giants science return from this advancement would be very large, because it would enhance the science return of a primary probe (carrying a mass spectrometer) and provide ground truth for breaking degeneracies affecting remote-sensing measurements. The Mars science return could distinguish between seasonal and inter-annual variability, and reconcile puzzling differences between measurements from ground-based facilities and multiple spacecraft. The relevant questions of giant planet weather and climate in the ice giants, and surface-atmosphere interactions and habitability for Mars.

TABLE 2 - Nominal sensitivity requirements for composition measurements in Uranus in the 0.5-10 bar range (Sayanagi et al. 2020). Capability gap is relative to Table 1.

\begin{tabular}{|l|l|l|}
\hline Target gas & Sensitivity range (concentration) & Improvement needed (Uranus) \\
\hline $\mathrm{CH}_{4}$ & $1 \mathrm{ppm}$ to $5 \%$ & $10 \mathrm{x}$ \\
\hline $\mathrm{NH}_{3}$ & $10 \mathrm{ppb}$ to $0.1 \%$ & $250 \mathrm{x}$ \\
\hline $\mathrm{H}_{2} \mathrm{~S}$ & $10 \mathrm{ppb}$ to $0.1 \%$ & none \\
\hline $\mathrm{H}_{2} \mathrm{O}$ & $<100 \mathrm{ppb}$ & $1000 \mathrm{x}$ \\
\hline $\mathrm{CO}$ & $<100 \mathrm{ppb}$ & $5 \mathrm{x}$ \\
\hline
\end{tabular}

Development priority: instrument development. Most chip-based sensors in use today are cost-effective implementations for terrestrial applications. For planetary missions, sensors must be integrated with flight-capable electronics, hardened for the space environment, and coupled with gas-sample delivery systems. Radiation hardening may affect sensitivity.

Development priority: instrument and resource discoverability. Development of new technologies like chip-based composition sensors would be accelerated by NASA tools to 
enhance the discovery of instruments (both flown and under development) and resources needed for developing new instruments (such as atmosphere simulators / testing chambers). The IPM database (ipmdatabase.webnode.com) is a good example of a tool for instrument discovery. Planetary atmosphere simulators (testing chambers) appropriate for various solar system targets exist at locations including NASA Ames, JPL, GSFC, Johns Hopkins, Georgia Tech, and other locations. But the ability to discover these simulators, understand their capabilities, and initiate collaborations is limited by the lack of any tool indexing current facilities. Support for these tools could be organized similarly to a NASA PDS node.

\section{Recommendations}

We recommend that NASA specifically support studies to:

- increase the sensitivity of chip-based composition sensors to match measurement requirements in solar system atmospheric targets

- adapt sensors into complete instruments, with particular attention to flight electronics and sample delivery systems

Additionally, we recommend that NASA support tools that enhance the discoverability of instrumentation for solar system exploration, as well as resources for instrument development (such as atmosphere simulators for device testing).

\section{References}

Atreya, S.K. \& Z.G. Gu (1994) JGR 99, 13133.

Atreya, S.K. et al. (2020) SSRv 216, 18.

Bjoraker, G.L. et al. (2018) AJ 156, 101.

de Pater, I., P.N. Romani \& S.K. Atreya (1991) Icar 91, 220.

Fahad, H.M., et al. (2017) SciA 3, e1602557.

Folkner, W.M., R. Woo \& S. Nandi (1998) JGR 103, 22847.

Formisano, V. et al. (2004) Sci 306, 1758.

Giles, R.S. et al. (2017) Icar 289, 254.

Glaze, L.S. et al. (2018) SSRv 214, 89.

Guillot, T. et al. (2020) JGR 125, e2020JE006403.

Gulkis, S., M.J. Janssen \& E.T. Olsen (1978) Icar 34, 10.

Hannon, A. et al. (2014) J. Sens. Sens. Syst. 3, 349.

Hannon, A. et al. (2016) Sensors 16, 1163.

Hueso, R. \& Á. Sánchez-Lavega (2019) SSRv 215, 52.

Irwin, P.G.J. et al. (2018) Nat Astron 2, 420.

Irwin, P.G.J. et al. (2019) Icar 329, 550.

Johnson, N.M. \& M.R.R. de Oliveira (2019) E\&SS 6, 1299.

Korablev, O. et al. (2019) Nat 568, 517.

Krasnopolsky, V.A. (1993) Icar 101, 313.

Krasnopolsky, V.A., J.P. Maillard \& T.C. Owen (2004) Icar 172, 537.

LI, C. et al. (2017) GRL 44, 5317.

Li, J. et al. (2012) SENSORS, 2012 IEEE, link.
Li, J., et al. (2003) NanoL 3, 929.

Lu, Y., et al. (2004) CPL 391, 344.

Mahaffy, P.R., et al. (2013) Sci 341, 263.

Makel, D.B. et al. (2020) LPSC Abs. \#2326.

McConnochie, T. et al. (2019) AGU FM \#P41B-3420.

Moores, J. et al. (2020) Decadal Survey Response ID $\underline{151}$.

Mumma, M.J. et al. (2009) Sci 323, 1041.

Niemann, H.B. et al. (1998) JGR 103, 22831.

Niemann, H.B., et al. (1998) JGR 103, 22831.

Oyama, V.I., B. J. Berdahl, \& G. C. Carle (1977) Natur 265, 110.

Sayanagi, K.M., et al. (2020) SSRv 216, 72.

Sromovsky, L.A., P.M. Fry \& J.H. Kim (2011) Icar 215, 292.

Stamenkovic, V. et al. (2018) Nat Geo 11, 95.

Tollefson et al. (2019) AJ 157, 251.

Trainer, M.G., et al. (2019) JGR 124, 3000.

Veverka, J. (2010) NASA Tech Rpt SDO-12367.

Webster, C.R., et al. (2013) Sci 342, 355.

Webster, C.R., et al. (2018) Sci 360, 1093.

Wong, A.S., S.K. Atreya \& Th. Encrenaz (2003) JGR 108, 5026.

Wong, M.H., et al. (2004) Icar 171, 153.

Young, P. et al. (2005) J. Nano. Nano. 5, 1.

Yung, Y.L. et al. (2018) Astrobiology 18, 1221. 